\title{
A Mathematical Model for Supermarket Order Picking
}

\author{
Eliana Costa e Silva, Manuel Cruz, Isabel Cristina Lopes and Ana Moura
}

\begin{abstract}
Order picking consists in retrieving products from storage locations to satisfy independent orders from multiple customers. It is generally recognized as one of the most significant activities in a warehouse (Koster et al, 2007). In fact, order picking accounts up to 50\% (Frazelle, 2001) or even 80\% (Van den Berg, 1999) of the total warehouse operating costs. The critical issue in today's business environment is to simultaneously reduce the cost and increase the speed of order picking. In this paper, we address the order picking process in one of the Portuguese largest companies in the grocery business. This problem was proposed at the $92^{\text {nd }}$ European Study Group with Industry (ESGI92). In this setting, each operator steers a trolley on the shop floor in order to select items for multiple customers. The objective is to improve their grocery e-commerce and bring it up to the level of the best international practices. In particular, the company wants to improve the routing tasks in order to decrease distances. For this purpose, a mathematical model for a faster open shop picking was developed. In this paper, we describe the problem, our proposed solution as well as some preliminary results and conclusions.
\end{abstract}

Eliana Costa e Silva

CIICESI/ESTGF-IPP - Center for research and innovation business sciences and information systems / School of Management and Technology of Felgueiras / Porto Polytechnic, Portugal, e-mail: eos@estgf.ipp.pt

Manuel Cruz

LEMA/ISEP/IPP - Mathematical Engineering Lab / School of Engineering / Porto Polytechnic, Portugal, e-mail: mbc@isep.ipp.pt

Isabel Cristina Lopes

LEMA/CIEFGEI/ESEIG-IPP - Mathematical Engineering Lab / School of Management and Industrial Studies/ Porto Polytechnic, Portugal, e-mail: cristinalopes@eseig.ipp.pt

Ana Moura

LEMA/ISEP/IPP - Mathematical Engineering Lab / School of Engineering / Porto Polytechnic and CMUP - Center of Mathematics, University of Porto, Porto, Portugal and CMUP, e-mail: aim@isep.ipp.pt 


\section{Introduction}

This paper addresses the problem of the order picking process on large grocery e-commerce business. This is a case study of one of the major players in the Portuguese grocery business, SonaeMC, which was presented to the $92^{\text {nd }}$ European Study Group with Industry (ESGI92). It regards the optimization of the order picking process that is conducted in an open shop, to fulfill orders from online customers. The challenge proposed to the participants of the ESGI92 was to boost the efficiency in the picking rate in $10 \%$. Optimizing the order picking process for faster picking and $100 \%$ accuracy (as a goal) is crucial to increase the competitiveness of the company and to preserve customers. This work includes several ideas held by the contributors of the ESGI92, as well as the modeling, implementation, some tests and improvements made afterwards by the authors of this paper.

Order picking may be defined as the process of retrieving products from storage in response to a specific customer request. It is generally recognized as one of the most significant activities in a warehouse. According to different authors, order picking accounts up to 50\% [4], or even $80 \%$ [9], of the total warehouse operating costs. The picker's time distribution has been estimated to be around $50 \%$ for traveling, $20 \%$ for searching and $15 \%$ for picking, with the remaining $15 \%$ being divided between setups and other minor activities [7]. In this paper, we focus our efforts on the third and fourth of the following main issues of the order picking activity [6]:

1. Storage Assignment - The assignment of articles to storage locations.

2. Zoning - The establishment of work zones to which pickers are confined.

3. Order Consolidation - The transformation of customer orders into picking orders.

4. Routing - The determination of sequences (routes) according to which the items have to be picked.

We propose a mathematical model to be solved with integer linear programming (ILP) techniques. The goal is to minimize the distance traveled by the pickers inside the shop. At first sight, this may resembles a capacitated vehicle routing problem (CVRP). However, for a given vehicle (picking trolley), the items from each customer order must be grouped in the proper boxes, which poses additional constraints to the problem. The CVRP is already a NP-hard problem. Furthermore, given the large number of orders processed by SonaeMC daily, the optimization problem is a large scale one. The need to get the solution in a small period of time and to deal with the additional constraints, led us to propose a tailored model (see Section 3).

\section{An overview of the company's picking process}

In this section we present an overview of the company's picking process at the time of the ESGI92. SonaeMC holds, all over Portugal, stores with different dimensions 
and architectures. However, they all share the topology depicted in Fig. 1, where five areas can be found:

- The shop - where the customers that visit the store pick their products.

- The warehouse - where the products are stored for replacement.

- The depot - an area inside the warehouse where the products of each client are gathered and packed.

- The HRP area - an area inside the warehouse where the High Rotation Products are stored.

- The dock - where the packed orders are loaded into vans that will deliver them to their final destination (the customer's address).

Fig. 1 Each store consists of five different areas: the shop, the warehouse, the depot, high rotation products area (HRP) and the dock.

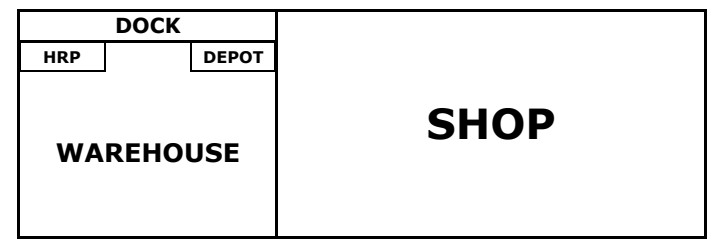

The process of assembling an order may be described as follows. An online customer makes an order on the company's website. The e-commerce team partitions the order in: High Rotation Products (HRP) - top selling articles or seasonal products; Fresh Products (FP) - products with special temperature and/or conservation requirements; and Regular Products (RP) - all the other products. The RP items are collected inside the shop by pickers who take them to the depot, where they are gathered with the HRP items that were previously packed for that customer. The remaining products - FP - are only delivered (by another picker) at the dock station when the order is taken to the van that will make the deliver.

We devoted our attention to the Regular Products (RP) which, according to the company, is the largest subset of items and where the pickers spend most of the time.

Unlike a warehouse, a supermarket is not designed to be efficient for the picking process (the goal is the opposite: make the customer see as much as possible). Therefore, changing the location of the products was not allowed.

At that time, pickers collected items for a single customer at a time. The purpose for this was to minimize the probability of swapping products between different orders. However, the picking trolleys have several separate boxes, which allows picking for multiple clients, and as they are already equipped with barcode scanners, the swapping can be avoided with a simple software update (checking in which box the item was placed). 


\section{The Model}

The problem may be stated as: Given a set of orders of different customers, the objective is to minimize the traveling distance needed to pick all the items, satisfying the weight and volume capacity constraints of the trolley, and imposing that in each box there are items of only one customer.

For the capacity constraints, let $R$ be the maximum number of routes allowed, $B$ the number of boxes per trolley, $W$ the maximum weight allowed per box and $S$ the maximum size allowed per box.

Let $I_{c}$ be the set of items requested by customer $c \in C$ and $I=\cup_{c} \in C I_{c}$ the set of items to be picked. An item is understood as the quantity of a specific product ordered by one customer, i.e., in our model, the same product requested by different customers produces different items. For all $i \in I$, let $w_{i}$ and $s_{i}$ be, respectively, the weight and the size of item $i$, and $c_{i}$ the customer who requested it. We suppose that $w_{i} \leq W$ and $s_{i} \leq S$, for all $i \in I$. If not, we previously divide the whole quantity of a specific product by a sufficient number of items in order to satisfy the conditions.

Our problem can be viewed as a variant of the CVRP Problem (see [8] for some variants), where:

- the depot $d$ and each aisle $v_{i}$ in the supermarket belong to the set of vertices $V$;

- each circuit (route) visits the depot vertex $d$ exactly once;

- each vertex is visited at least once in the total of the circuits, and at most once in each circuit;

- each vertex $v_{i}$ needs to be visited while there are items $i$ to collect there;

- the total weight and the total volume of the items picked in a circuit do not exceed the vehicle and boxes capacity;

- the items are separated according to clients as they are being picked and put in the boxes of the trolley respecting the assignment of boxes to clients.

We construct a directed weighted graph $G=\left(V_{I}, A, \rho\right)$ as follows (see Fig. 2). For every $i \in I$, the set $V_{I}$ is the subset of $V$ consisting of vertices $v_{i} \in V$ corresponding to the aisles where items $i$ are stored, together with the depot. Note that while $V$ consists of all aisles of the shop (and the depot), $V_{I}$ contains only the aisles with the items to be picked (and the depot). To define the set of arcs $A$, we consider a total ordering on the set of aisles, without the depot, $V_{I} \backslash\{d\}$, defined as:

$$
u<v \text { if and only if } u_{x}<v_{x} \vee\left(u_{x}=v_{x} \wedge u_{y}>v_{y}\right),
$$

where $\left(u_{x}, u_{y}\right)$ and $\left(v_{x}, v_{y}\right)$ are the coordinates of the center of the aisles $u, v \in$ $V_{I} \backslash\{d\}$. Now, the $\operatorname{arcs} A$ of the graph are:

$$
A=\left\{(u, v) \in\left(V_{I} \backslash\{d\}\right)^{2} \mid u<v\right\} \cup\left\{(u, v) \in V_{I}^{2} \mid u=d \vee v=d\right\} .
$$

Finally, the function $\rho: A \rightarrow \mathbb{R}$ defines the weight of every arc $(u, v) \in A$ as the minimum distance that the picker needs to travel throughout the aisles of the store, to get from vertex $u$, located at the center of an aisle, to vertex $v$, at the center of another 

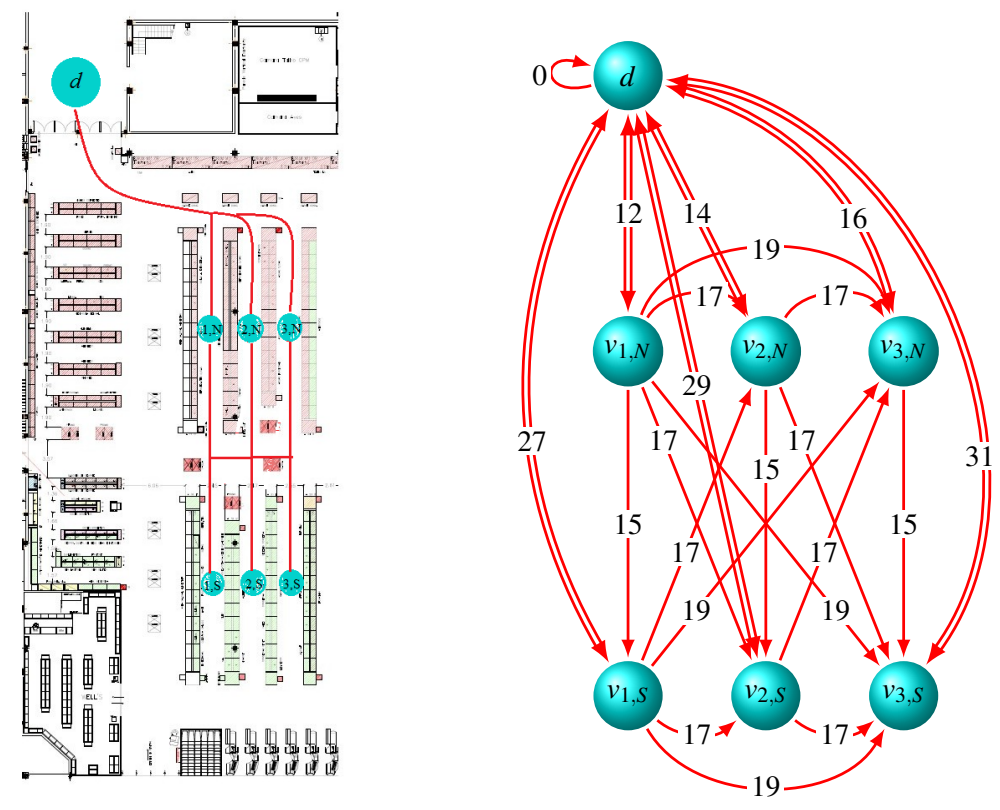

Fig. 2 An example of the directed graph $G$ of part of the store, with the depot $d$, and aisles $v_{1, N}, v_{1, S}, v_{2, N}, v_{2, S}, v_{3, N}$ and $v_{3, S}$. The labels of the arcs are the distances.

aisle (it is not the usual euclidean distance). To formulate the problem we use the binary variables $x_{u v}^{r}=1$ if arc $(u, v) \in A$ is selected in route $r$, with $r=1, \ldots, R$, and $y_{i}^{r b}=1$ if item $i \in I$ is picked in route $r$ into box $b$, with $r=1, \ldots, R$, and $b=1, \ldots, B$. The model is the following.

$$
\begin{array}{lll}
\text { Min } & \sum_{1 \leq r \leq R} \sum_{(u, v) \in A} \rho_{u v} x_{u v}^{r} & \\
\text { s.t. } & \sum_{(v, u) \in A} x_{v u}^{r} \leq 1 & \forall r=1, \ldots, R, \forall v \in V_{I} \backslash\{d\} \\
& \sum_{(u, v) \in A} x_{u v}^{r} \leq 1 & \forall r=1, \ldots, R, \forall v \in V_{I} \backslash\{d\} \\
& \sum_{(v, u) \in A} x_{v u}^{r}=\sum_{(u, v) \in A} x_{u v}^{r} \quad \forall r=1, \ldots, R, \forall v \in V_{I} \backslash\{d\} \\
& \sum_{r=1, \ldots, R} \sum_{(v, u) \in A} x_{v u}^{r} \geq 1 & \forall v \in V_{I} \backslash\{d\} \\
& \sum_{r=1, \ldots, R} \sum_{(u, v) \in A} x_{u v}^{r} \geq 1 & \forall v \in V_{I} \backslash\{d\}
\end{array}
$$




$$
\begin{aligned}
\sum_{(d, u) \in A} x_{d u}^{r}=1 & \forall r=1, \ldots, R \\
\sum_{(u, d) \in A} x_{u d}^{r}=1 & \forall r=1, \ldots, R \\
\sum_{r=1, \ldots, R} \sum_{b=1, \ldots, B} y_{i}^{r b}=1 & \forall i \in I \\
\sum_{b=1, \ldots, B} y_{i}^{r b} \leq \sum_{\left(u, v_{i}\right) \in A} x_{u v_{i}}^{r b} & \forall r=1, \ldots, R \forall i \in I \\
y_{i}^{r b}+y_{j}^{r b} \leq 1 & \forall i \in I, \forall j \in I \backslash I_{c_{i}}, \forall r=1, \ldots, R, \forall b=1, \ldots, B \\
\sum_{i \in I} w_{i} y_{i}^{r b} \leq W & \forall r=1, \ldots, R, \forall b=1, \ldots, B \\
\sum_{i \in I} s_{i} y_{i}^{r b} \leq S & \forall r=1, \ldots, R, \forall b=1, \ldots, B \\
x_{u v}^{r b} \in\{0,1\} & \forall(u, v) \in A, \forall r=1, \ldots, R \\
y_{i}^{r b} \in\{0,1\} & \forall i \in I, \forall r=1, \ldots, R, \forall b=1, \ldots, B
\end{aligned}
$$

The objective function in (1) deems to minimize the total traveling distance. Constraints (2) and (3) ensure that there will be at most one arc leaving every aisle $v$, and there will be at most one arc entering $v$ in each route $r$, for all vertices in $V_{I} \backslash\{d\}$. Equations (4) ensure that, for each route, the number of arcs entering an aisle is equal to the number of arcs leaving it. Constraints (5) and (6) guarantee that each aisle is visited at least once in the total of routes. There are also similar constraints for the depot: (7) and (8). The next constraints concern the boxes where the items are placed. In (9) we force that each item is picked to exactly one box in exactly one route. Also, if an item is picked in a route, its aisle needs to be visited in such route; this is given by (10). Inequalities (11) guarantee that each box in a route does not have items of different customers. Finally, we add the weight and volume constraints concerning the dimensions of the boxes (12) and (13). The range of the variables are established in (14) and (15).

\section{Results}

The model was implemented using AMPL [3] and tested with real data provided by SonaeMC, regarding the orders placed at one of their main stores in a given period. The dataset was composed of several orders, with an overall weight of $\approx 600 \mathrm{~kg}$ and volume of $\approx 1,6 \mathrm{~m}^{3}$. In our implementation we set $R=10, B=6, W=12 \mathrm{~kg}$ and $S=46400 \mathrm{~cm}^{3}$. The model was submited to Gurobi solver on NEOS online server $[1,2,5]$.

As the complexity of this kind of model is non-polynomial, we used an heuristic to reduce the dimension of the problem. This heuristic computes a matrix of distances between customers, based on their similarity in terms of number of products in common aisles, and gathers the orders in clusters, so that the available compu- 
tational resources may solve the integer programming model, within these clusters. The heuristic splits our set of orders in 7 subsets: 3 of them with products in a single aisle (no routing needed), and 4 clusters with products in several aisles. For each one of these 4 clusters, we solved the IP model in (1)-(15). In this 4 subsets the optimal solutions were found, in a total time of 435 seconds.

We compared the results of our model with a simulation of SonaeMC's current picking. We were able to reduce the total distance by $39 \%$, from 1341.5 to 818 meters. The number of routes and the number of boxes is similar in both. With our routing solution, the picker would increase the picking rate in $24 \%$.

\section{Conclusions}

We focused on a case study of a large Portuguese grocery company, regarding the open shop order picking process for online customers. We developed an integer programming model for a variant of the Capacitated Vehicle Routing Problem, with additional constraints to deal with the specificity of the trolley and the company's requirements. The model integrates the batching and the routing problems with very promising first results. Also, an heuristic was designed for reducing the dimension of the problem, in order to obtain reasonable computational times. On a real dataset, the model reduced the total traveling distance by $39 \%$, and increased the picking rate in $24 \%$, a result that exceeded the company's expectations.

Acknowledgements A. Moura was partially supported by CMUP (UID/MAT/00144/2013), which is funded by FCT (Portugal) with national (MEC) and European structural funds through the programs FEDER, under the partnership agreement PT2020.

\section{References}

1. Czyzyk, J., Mesnier, M., Moré,J.: The NEOS Server. In: IEEE Journal on Computational Science and Engineering 5 (3), pp. 68-75. (1998)

2. Dolan,E.: The NEOS Server 4.0 Administrative Guide, Technical Memorandum ANL/MCSTM-250. Mathematics and Computer Science Division, Argonne National Laboratory (2001).

3. Fourer, R., Gay,D. M. and Kernighan,B. W.: AMPL - a modeling language for mathematical programming. Thomson/Brooks/Cole, Pacific Grove, CA, (2003)

4. Frazelle, E.: World-Class Warehousing and Material Handling. McGraw-Hill, New York (2001)

5. Gropp, W., Moré, J.: Optimization Environments and the NEOS Server. In: M. D. Buhmann, A. Iserles (eds.) Approximation Theory and Optimization, pp. 167-182. Cambridge University Press (1997).

6. De Koster, R., Le-Duc, T. and Roodbergen, K. J.: Design and control of warehouse order picking: a literature review. In: European Journal of Operational Research 182 (2), pp. 481-501 (2007)

7. Tompkins, J., White, J., Bozer, Y., and Tanchoco, J.: Facilities planning Wiley, New Jersey (2003) 
8. Toth, P. and Vigo, D.: The Vehicle Routing Problem In: Monographs on Discrete Mathematics and Applications, Society for Industrial and Applied Mathematics (2002)

9. Van den Berg,J. P.: A literature survey on planning and control of warehousing systems In: IIE Transactions 31 (8), pp. 751-762 (1999) 